\title{
Deep Vein Thrombosis in the Uninjured Limbs of Lower Extremity Fractures: A Retrospective Study of 1454 Patients
}

\section{Shuang-Wei Qu}

Xi'an Jiaotong University

\section{Yu-Xuan Cong}

Xi'an Jiaotong University

\section{Peng-Fei Wang}

Xi'an Jiaotong University

Chen Fei

Xi'an Jiaotong University

Zhi Li

Xi'an Jiaotong University

\section{Kun Yang}

Xi'an Jiaotong University

Kun Shang

Xi'an Jiaotong University

Chao Ke

Xi'an Jiaotong University

Hai Huang

Xi'an Jiaotong University

Yan Zhuang

Xi'an Jiaotong University

Bin-Fei Zhang ( $\square$ zhangbf07@gmail.com )

Xi'an Jiaotong University https://orcid.org/0000-0002-1135-6972

Kun Zhang

Xi'an Jiaotong University

\section{Research}

Keywords: deep vein thrombosis, lower extremities fractures, ultrasonography, uninjured limb

Posted Date: October 9th, 2020

DOl: https://doi.org/10.21203/rs.3.rs-88284/v1 
License: (c) (i) This work is licensed under a Creative Commons Attribution 4.0 International License. Read Full License 


\section{Abstract}

Objective: The purpose of this study was to investigate the incidence of deep venous thrombosis (DVT) in the uninjured limb, before and after operation, in patients with lower extremity fractures.

Methods: We collected the clinical data of patients with lower extremities fractures who presented at Xi'an Honghui Hospital between 1 July, 2015 and 31 October, 2017. Doppler ultrasonography was used to diagnose the DVT. Patients were examined pre-and postoperatively. The patients were then divided into a thrombosis group and a no thrombosis group according to the preoperative and postoperative ultrasonography results. The thrombosis group was defined as patients admitted to our hospital with DVT in the uninjured limb and the no thrombosis group was defined as patients without DVT in the uninjured limb.

Results: This study enrolled 1454 patients who met the inclusion criteria. The incidence of preoperative DVT in the uninjured limb was $9.63 \%$ whereas the postoperative incidence was $20.29 \%$. Age (OR $=0.965$, $95 \mathrm{Cl} \%$ : $0.954-0.977 ; P \leq 0.001)$ and gender (OR=0.667, 95\% Cl: 0.451-0.986, $P=0.042)$ were independent risk factors for preoperative DVT in the uninjured limb. Blood loss (OR=0.997, $95 \mathrm{Cl} \%$ : 0.995-1.000; $P=0.020$ ), $D$-dimer levels at admission ( $\mathrm{OR}=0.941,95 \mathrm{Cl} \%$ : 0.887-0.999; $P=0.045$ ), and postoperative day 5 D-dimer levels (OR=0.889, $95 \mathrm{Cl} \%$ : 0.819-0.965; $P=0.005)$, were independent risk factors for postoperative DVT in the uninjured limb.

Conclusion: In conclusion, the actual incidence of DVT in the uninjured lower extremity after fracture may currently be underestimated and should be pay more attention.

\section{Introduction}

Deep vein thrombosis (DVT) occurs frequently in orthopaedic patients. It can increase the length of the hospital stay and associated costs, affect the recovery process of patients, and potentially cause a fatal pulmonary embolism [1]. In recent years, orthopaedists have paid increasing attention to the prophylaxis and treatment of DVT. Currently, drug prevention is considered one of the most effective methods to decrease the risk of lower extremity DVT [2]. Traditional low-molecular-weight heparin (LMWH) [3] is used as the standard protocol.

There are only a few studies that directly identify the incidence and importance of DVT of the uninjured limb in patients with lower extremity fractures. Mok et al. reported that the incidence of DVT in uninjured limbs is $14.3 \%$ [4].Song et al. reported that $29.4 \%$ of preoperative patients had DVT in either the uninjured or injured limb and that percentage has increased to $32.8 \%$ postoperatively[5]. In fact, there was a risk for DVT in the uninjured limb as well. Meissner et al suggested that the hypercoagulation state persists for at least one month after injury in $80 \%$ of patients [6]. Therefore, DVT in the uninjured limb is possible. Decker and Weaver et al. demonstrated that a DVT could occur in both the injured and uninjured leg with a trend for higher incidences in the injured leg [7]. 
On the basis of these factors, this study aimed to retrospectively analyse the data of patients with lower extremity fractures to investigate the incidence of DVT in the uninjured limb in in-patients with lowerextremity fractures.

\section{Patients And Methods}

\section{Ethical statement}

The study was approved by the Institutional Review Board (IRB) of Xi'an JiaoTong University.

\section{Included and excluded criterion}

Inclusion criteria: a) age $\geq 16$ years, b) recent lower extremity fractures requiring surgical treatment, c) hospital stay $>48 \mathrm{~h}, \mathrm{~d}$ ) availability of results of preoperative and postoperative ultrasonography. Exclusion criteria: a) delayed lower extremity fractures, defined as injuries without surgical treatment within 3 weeks of injury, b) open soft tissue fractures $c$ ) serious medical problems without tolerating surgery, d) poor compliance patients.

\section{Methods}

All patients admitted to the hospital were routinely assessed using the the risk assessment profile for thromboembolism score (RAPT) to determine the risk of thromboembolism. We also assessed patients for contraindications for anticoagulation therapy. For patients without anticoagulation contraindications, LMWH (3800 IU/0.4 mL once per day, Fraxiparine; Glaxo Wellcome Production, GlaxoSmithKline) was injected subcutaneously to prevent DVT. Mechanical thrombo-prophylaxis, administered for 20 min, twice a day, via a pressure pump was also utilized to prevent a DVT. Blood samples were collected within two hours after admission, one day preoperatively, one day postoperatively, and five days postoperatively.

We used Doppler ultrasonography to diagnose the DVT. The diagnostic criteria the presence of a constant intraluminal filling defect. Patients were examined pre-and postoperatively. All patients underwent ultrasonography of the double lower limbs on the day before the scheduled surgery and between the third and fifth day after surgery. DVTs were classified into three types: central (femoral and iliac veins), peripheral (calf muscle, fibular, and anterior/posterior tibial veins), and mixed thrombosis (both central and peripheral thromboses).

Based on the results of the ultrasonography, the patients were divided into two groups: thrombosis group and no thrombosis group. The thrombosis group was defined as patients admitted to our hospital suffering a DVT in the uninjured limb and the no thrombosis group was defined as patients without a DVT in the uninjured limb. For patients without thrombosis, LMWH (3800 IU/0.4 mL once per day, Fraxiparine; Glaxo Wellcome Production, GlaxoSmithKline) was continuously injected subcutaneously to prevent DVT. Patients with thrombosis had LMWH (3800 IU/0.4 mL, twice per day) injected subcutaneously to treat the DVT. When preoperative ultrasonography results showed a central or mixed thrombosis, an inferior vena cava filter was used to prevent the risk of pulmonary embolism. The 
anticoagulant therapy was stopped 12 hours before surgery and restarted again 24 hours after surgery. When the patients were discharged, the protocol was changed to rivaroxaban for 35 days after operation.

\section{Statistics analysis}

Statistical analysis was performed using the SPSS Version 19.0 (SPSS Inc., Chicago, Illinois, USA). The measurement data was analyzed to determine if the data is normally distributed. The statistical analysis utilized the independent sample t-test. For the enumeration data, chi-square test was used. Multivariate analysis was used to detect the risk factors. The difference was statistically significant if $P<0.05$.

\section{Results}

\section{Patient characteristics}

In total, 1454 patients suffering a lower extremity fracture were screened. After excluding, 334 patients suffering uninjured limb DVT. Of these patients, 269 fractures happen above the knee, 37 fractures happen around the knee, 28 fractures happen below the knee. All the patients underwent surgery. The procedures included: open reduction with internal fixation in 284 patients, hemiarthroplasty in 3 patients, total hip replacement in 1 patient, and closed reduction in 46 patients. All patients were treated with lowmolecular-weight heparin at admission. On average, all the patients were aged 71.43 (standard deviation [SD] 13.86) years range from 17 to 95 years old. Of these patients, $218(65.27 \%)$ were female and116 $(34.73 \%)$ were male. No fatal bleeding events or bleeding into a critical site was found in this study.

\section{The incidence of uninjured limb DVT in preoperative ultrasound}

The preoperative ultrasound data for patients with a lower extremity fracture with or without a thrombosis in the uninjured limb was compared in the patients' characteristics (Table 1). All the patients were diagnosed busing ultrasound to identify the thrombosis in lower limbs. There were 140 patients with a thrombosis to the uninjured limb $(9.63 \%)$ and 1314 patients without a thrombosis to the uninjured limb (90.37\%). There were $9.01 \%$ peripheral DVT, $0.07 \%$ central DVT, and $0.55 \%$ mixed DVT. Peripheral DVT constituted $93.57 \%(131 / 140)$ of all DVTs.

Importantly, there were 50 patients with a left lower extremity fracture who suffered a DVT in both lower extremities (3.44\%), 22 with a left lower extremity fracture who suffered a right lower extremity DVT $(1.51 \%), 34$ with a right lower extremity fracture who suffered a left lower extremity DVT $(2.34 \%)$ and 34 with right lower extremity fractures who suffered a DVT in both lower extremities (2.34\%).

\section{Univariate analysis of preoperative uninjured limb DVT}

There were no statistically significant differences between the two groups in: medical morbidity excluding hypertension and coronary heart disease, Body Mass Index, days between fracture and hospitalization, Ddimer at admission, preoperative D-dimer, or CRP at admission. However, in the thrombosis group, female patients, patients with a fracture located above the knee, and patients classified as stage $\nabla$ of American 
Society of Anesthesiologists Physical Status Classification System (ASA) were statistically different than the no thrombosis group. In addition, more patients in the thrombosis group suffered hypertension and coronary heart disease than in the no thrombosis group. (32.86\% vs. $21.91 \%, P=0.003 ; 32.86 \%$ vs. $21.76 \%, P=0.003)$, Age ( $72.42 \pm 13.57$ vs. $60.98 \pm 19.38, P \leq 0.001)$ was found to be another vital factor contributing to thrombosis (Table 1 ).

\section{Multivariate analysis of preoperative uninjured limb DVT}

Multivariate analysis was used to detect the risk factors by entering age, sex, types of fracture, hypertension, coronary heart disease and ASA classification. The results showed that age $(\mathrm{OR}=0.965,95$ $\mathrm{Cl} \%$ : 0.954-0.977; $P \leq 0.001)$ and sex $(\mathrm{OR}=0.667,95 \% \mathrm{Cl}: 0.451-0.986, P=0.042)$ were independent risk factors for preoperative DVT.

\section{The incidence of uninjured limb DVT in postoperative ultrasound}

All patients underwent ultrasonography postoperatively to identify the thrombosis in lower limbs.

Thrombosis was found in the uninjured limb of 295 patients $(20.29 \%)$ and 1159 patients did not have a thrombosis in the uninjured limb (79.71\%). There were $19.33 \%$ peripheral DVT, $0.21 \%$ central DVT, $7.57 \%$ mixed DVT. Peripheral DVT constituted 95.25\% (281/295) of all DVTs (Table 2).

Importantly, there were 127 patients with a left lower extremity fracture who suffered a DVT in both lower extremities (8.73\%), 25 with a left lower extremity fracture who suffered a DVT in the right lower extremity (1.72\%), 33 with a right lower extremity fracture who suffered a DVT in the left lower extremity $(2.27 \%)$ and 110 with a right lower extremity fracture who suffered a DVT in both lower extremities $(7.56 \%)$.

\section{Univariate analysis of postoperative uninjured limb DVT}

In two groups, there was no statistically significant difference in the medical morbidity excluding hypertension, coronary heart disease and stroke, Body Mass Index, length of hospital stay, days between fracture and hospitalization, days between fracture and operation, duration of operation, liquid transfusion, preoperative D-dimer values, C-reactive protein at admission and at postoperative day 5 . However, in the thrombosis group, more patients were female $(P \leq 0.001)$, fracture above the knee $(P \leq$ $0.001)$, and ASA stage $\mathbb{\nabla}(P \leq 0.001)$ compared to the no thrombosis group. Similar to the preoperative ultrasound data, age is a contributing factor to postoperative thrombosis $(71.76 \pm 13.42$ vs. $59.62 \pm 19.66$, $P \leq 0.001)$. Coronary heart disease, stroke, and hypertension were also important factors contributing to postoperative thrombosis $(P=0.003, P=0.017, P=0.009)$. In addition, blood loss was another factor that contributed to postoperative thrombosis $(P \leq 0.001)$. In the serum markers, D-dimer at admission, at postoperative day 1 , at postoperative day 3 , at postoperative day 5 were higher in the thrombosis group (Table 2).

\section{Multivariate analysis of postoperative uninjured limb DVT}


Multivariate analysis was used to detect the risk factors by entering age, gender, types of fracture, hypertension, coronary heart disease, stroke, ASA classification, blood loss, D-dimer at admission, Ddimer at postoperative day 1, D-dimer at postoperative day 3 and at D-dimer at postoperative day 5 . The results show that $\mathrm{D}$-dimer at admission ( $\mathrm{OR}=0.941,95 \mathrm{Cl} \%$ : 0.887-0.999; $P=0.045)$, $\mathrm{D}$-dimer at postoperative 5 day (OR $=0.889,95 \mathrm{Cl} \%$ : 0.819-0.965; $P=0.005$ ), and blood loss (OR $=0.997,95 \mathrm{Cl} \%$ : 0.995-1.000; $P=0.020$ ) were independent risk factors for postoperative DVT.

\section{The dynamic changes of uninjured limb DVT before and after operation}

There were 334 patients suffering uninjured limb DVT in 1454 patients. There was a total of $53.60 \%$ $(0.90 \%, 52.40 \%, 0.30 \%)$ patients without changes during the hospital stay. There were $5.39 \%$ of patients who went from having a preoperative peripheral DVT to no thrombosis postoperatively. Conversely, $34.73 \%(33.83 \%, 0.30 \%, 0.60 \%)$ of patients changed from having no preoperative thrombosis to developing a peripheral, central, or mixed DVT postoperatively (Fig. 1).

\section{Discussion}

There are few studies that directly identify the incidence and importance of DVT in the uninjured limb in patients with lower extremity fractures. Most previous research analysed the distribution of DVT in the injured limb across the different fractures[3], or risk factors contributing to a DVT in the injured limb during the hospital stay[8]. Song et al [5] reported the changes in DVT in the injured limb in 119 patients and found a high incidence of preoperative DVT in patients[5]. They also found that the incidence of DVT in the uninjured limb is low. In addition, some studies reported symptomatic DVT but ignored asymptomatic DVT. Sun et al. reported that $11.2 \%$ of DVTs are asymptomatic[9]. Some studies reported that all patients suffering DVT were clinically asymptomatic [10, 11]. In the field of thrombosis after fracture, we should not only focus on symptomatic DVT but also pay attention to asymptomatic DVT. Asymptomatic DVT is a potentially important but easily ignored risk factor for thrombosis.

We retrospectively investigated the changes in preoperative, postoperative, symptomatic, and asymptomatic DVTs in the uninjured limb of patients with lower extremity fractures, We found: $\mathbf{a}$. the incidence of preoperative DVT in the uninjured side is $9.63 \%$ and increases to $20.29 \%$ postoperatively, $\mathbf{b}$. peripheral DVT constitutes $93.57 \%$ of all preoperative and $95.25 \%$ of postoperative uninjured limb DVTs.

The dynamic changes in preoperative and postoperative uninjured limb DVT are obvious. There were 116 new postoperative DVTs to the uninjured limb, primarily peripheral DVTs. Peripheral DVTs are likely to occur in calf muscle veins[12]. The DVTs in the uninjured limb of 298 patients were peripheral from before to after operation. The result is similar to DVT of the injured limb. The majority of patients diagnosed with a DVT in the uninjured limb postoperatively had already suffered a DVT in the uninjured limb before operation[5].

In this study, the incidence of DVT in the uninjured limb in lower extremity fractures is high, increasing from $9.63 \%$ preoperatively to $20.29 \%$ postoperatively. Previous studies report that the incidence is lower 
than that in our study but the reasons vary[4]. First, some studies reported symptomatic DVT but ignored asymptomatic DVT. In fact, Sun and Chen et al. reported that $11.2 \%$ of DVTs are asymptomatic[9]. Second, the mean number of days between fracture and surgery is 6.95 days in our study. However, other studies have shown the preoperative incidence of injured limb DVT to be between $54 \%$ and $62 \%$ for patients with acute fracture whose surgery was delayed by $>48$ hours $[13,14]$. Most of the patients in this study presented a few days after the fracture occurred. The delay may result from preoperative medical evaluation and optimization or transfer from community hospitals to tertiary-care facilities. Elderly patients tend to be sicker on admission and therefore more likely to require more time for preoperative preparation $[8,9]$. The delays in surgical intervention may predispose patients to developing thromboembolic problems and may be related to the uninjured limb thromboembolic problems. Third, thrombosis could be detected by ultrasonography, venography, and multidetector computed tomographic venography $[8,15]$. The diagnostic sensitivity and the specificity is different for each modality and thus, these factors may be contributing to the high incidence of DVT more than other factors[15].

There are many factors contributing to the formation of thrombosis after trauma, including the fracture and surgical intervention[5, 16-18]. In this study, we found that age and gender were independent risk factors for preoperative DVT in uninjured limb and that D-dimer at admission and at postoperative day 5 and blood loss were independent risk factors for postoperative DVT. Knudson and Gomez et al. found that age is an independent risk factor for thrombosis in adult patients with trauma[19]. One study showed that the incidence of DVT is higher in men than women but the reasons are still not clear.[20] However, some scholars hold the opposite view, believing that gender does not affect the incidence of DVT[21]. Furthermore, blood loss is another factor promoting the hypercoagulability states[22]. In the multivariate analysis of postoperative DVT, blood loss is an independent risk factor $(\mathrm{OR}=5.430, P=0.020)$. Blood loss in thrombosis group is higher than in the no thrombosis group (Table 2); however, there is no difference in liquid transfusion. The hypercoagulation states, one factor that increases the thrombosis risk, may be the main reason contributing to this phenomenon. Meissner, Chandler et al. and Selby, Geerts et al. proved that the hypercoagulability state persists for a long time[6, 23]. In addition, the surgical intervention itself is the introducing factor for hypercoagulability states[24, 25]. Thus, DVT in uninjured limb and the increased incidence of DVT are easy to comprehend.

In conclusion, the actual incidence of DVT in the uninjured lower extremity after fracture may currently be underestimated and should be pay more attention.

\section{Abbreviations}

DVT Deep vein thrombosis

LMWH Low-molecular-weight heparin

BMI Body mass index

ASA American Society of Anesthesiologists 
OR Odds ratio

\section{Declarations}

\section{Acknowledgments}

We thank all our colleagues working in the Department of Orthopedic Trauma, Hong-Hui Hospital, Xi'an Jiaotong University.

\section{Funding}

This study was supported by the Social Development Foundation of Shaanxi Province (grant numbers 2017ZDXM-SF-009).

\section{Availability of data and materials}

The survey was implemented by Xi'an Honghui Hospital. According to relevant regulations, the data could not be shared.

\section{Author Contributions}

Conceptualization: ZY, ZK.

Data curation: FC, LZ, YK, KC, and HH.

Formal analysis: ZBF and QSW.

Roles/Writing-original draft: QSW, CYX and ZBF.

\section{Ethics approval and consent to participate}

The study was approved by the ethical board of Xi'an JiaoTong University (No. 2014026).

\section{Conflict of interest}

The authors declare that they have no competing interests.

\section{Consent for publication}

No

\section{References}

1. Brill JB, Badiee J, Zander AL, Wallace JD, Lewis PR, Sise MJ, Bansal V, Shackford SR: The Rate of Deep Vein Thrombosis Doubles in Trauma Patients with Hypercoagulable Thromboelastography. $J$ Trauma Acute Care Surg 2017, 83(3):413-419. 
2. Di Nisio M, van Es N, Buller HR: Deep vein thrombosis and pulmonary embolism. Lancet 2016, 388(10063):3060-3073.

3. Schick MA, Pacifico L: Deep Venous Thrombosis (DVT), Lower Extremity. In: StatPearls. edn. Treasure Island (FL); 2017.

4. Mok CK, Hoaglund FT, Rogoff SM, Chow SP, Ma A, Yau AC: The incidence of deep vein thrombosis in Hong Kong Chinese after hip surgery for fracture of the proximal femur. The British journal of surgery 1979, 66(9):640-642.

5. Song K, Yao Y, Rong Z, Shen Y, Zheng M, Jiang Q: The preoperative incidence of deep vein thrombosis (DVT) and its correlation with postoperative DVT in patients undergoing elective surgery for femoral neck fractures. Archives of orthopaedic and trauma surgery 2016, 136(10):1459-1464.

6. Meissner MH, Chandler WL, Elliott JS: Venous thromboembolism in trauma: a local manifestation of systemic hypercoagulability? J Trauma 2003, 54(2):224-231.

7. Decker S, Weaver MJ: Deep venous thrombosis following different isolated lower extremity fractures: what is known about prevalences, locations, risk factors and prophylaxis? Eur J Trauma Emerg Surg 2013, 39(6):591-598.

8. Shin WC, Woo SH, Lee SJ, Lee JS, Kim C, Suh KT: Preoperative Prevalence of and Risk Factors for Venous Thromboembolism in Patients with a Hip Fracture: An Indirect Multidetector CT Venography Study. J Bone Joint Surg Am 2016, 98(24):2089-2095.

9. Sun Y, Chen D, Xu Z, Shi D, Dai J, Qin J, Jiang Q: Incidence of symptomatic and asymptomatic venous thromboembolism after elective knee arthroscopic surgery: a retrospective study with routinely applied venography. Arthroscopy 2014, 30(7):818-822.

10. Smith EB, Parvizi J, Purtill JJ: Delayed surgery for patients with femur and hip fractures-risk of deep venous thrombosis. The Journal of trauma 2011, 70(6):E113-116.

11. Froehlich JA, Dorfman GS, Cronan JJ, Urbanek PJ, Herndon JH, Aaron RK: Compression ultrasonography for the detection of deep venous thrombosis in patients who have a fracture of the hip. A prospective study. J Bone Joint Surg Am 1989, 71(2):249-256.

12. Henry JC, Satiani B: Calf muscle venous thrombosis: a review of the clinical implications and therapy. Vascular and endovascular surgery 2014, 48(5-6):396-401.

13. Zahn HR, Skinner JA, Porteous MJ: The preoperative prevalence of deep vein thrombosis in patients with femoral neck fractures and delayed operation. Injury 1999, 30(9):605-607.

14. Hefley FG, Jr., Nelson CL, Puskarich-May CL: Effect of delayed admission to the hospital on the preoperative prevalence of deep-vein thrombosis associated with fractures about the hip. $J$ Bone Joint Surg Am 1996, 78(4):581-583.

15. Terao M, Ozaki T, Sato T: Diagnosis of deep vein thrombosis after operation for fracture of the proximal femur: comparative study of ultrasonography and venography. J Orthop Sci 2006, 11(2):146-153.

16. Park MS, Perkins SE, Spears GM, Ashrani AA, Leibson CL, Boos CM, Harmsen WS, Jenkins DH, Bailey $\mathrm{KR}$, Ballman KV et al: Risk factors for venous thromboembolism after acute trauma: A population- 
based case-cohort study. Thromb Res 2016, 144:40-45.

17. Park SJ, Kim CK, Park YS, Moon YW, Lim SJ, Kim SM: Incidence and Factors Predicting Venous Thromboembolism After Surgical Treatment of Fractures Below the Hip. J Orthop Trauma 2015, 29(10):e349-354.

18. Chen F, Xiong JX, Zhou WM: Differences in limb, age and sex of Chinese deep vein thrombosis patients. Phlebology 2015, 30(4):242-248.

19. Knudson MM, Gomez D, Haas B, Cohen MJ, Nathens AB: Three thousand seven hundred thirty-eight posttraumatic pulmonary emboli: a new look at an old disease. Annals of surgery 2011, 254(4):625632.

20. Kniffin WD, Jr., Baron JA, Barrett J, Birkmeyer JD, Anderson FA, Jr.: The epidemiology of diagnosed pulmonary embolism and deep venous thrombosis in the elderly. Arch Intern Med 1994, 154(8):861866.

21. Anderson FA, Jr., Spencer FA: Risk factors for venous thromboembolism. Circulation 2003, 107(23 Suppl 1):19-16.

22. Riha GM, Kunio NR, Van PY, Kremenevskiy I, Anderson R, Hamilton GJ, Differding JA, Schreiber MA: Uncontrolled hemorrhagic shock results in a hypercoagulable state modulated by initial fluid resuscitation regimens. J Trauma Acute Care Surg 2013, 75(1):129-134.

23. Selby R, Geerts W, Ofosu FA, Craven S, Dewar L, Phillips A, Szalai JP: Hypercoagulability after trauma: hemostatic changes and relationship to venous thromboembolism. Thromb Res 2009, 124(3):281-287.

24. Kupcinskiene K, Trepenaitis D, Petereit R, Kupcinskas J, Gudaityte R, Maleckas A, Macas A: Monitoring of Hypercoagulability by Thromboelastography in Bariatric Surgery. Med Sci Monit 2017, 23:1819-1826.

25. Wilson D, Cooke EA, McNally MA, Wilson HK, Yeates A, Mollan RA: Changes in coagulability as measured by thrombelastography following surgery for proximal femoral fracture. Injury 2001, 32(10):765-770.

\section{Tables}


Table 1

Patient characteristics according to preoperative ultrasound

\begin{tabular}{|c|c|c|c|c|}
\hline & Thrombosis & $\begin{array}{l}\text { No } \\
\text { Thrombosis }\end{array}$ & Overall & $P$ \\
\hline Number & 140 & 1314 & 1454 & \\
\hline Age & $\begin{array}{l}72.42 \pm \\
13.57\end{array}$ & $60.98 \pm 19.38$ & $\begin{array}{l}62.09 \pm \\
19.19\end{array}$ & $\begin{array}{l}\leq \\
0.001\end{array}$ \\
\hline \multicolumn{5}{|l|}{ Gender } \\
\hline Female & 98 & 694 & 792 & \multirow{2}{*}{$\begin{array}{l}\leq \\
0.001\end{array}$} \\
\hline Male & 42 & 620 & 662 & \\
\hline \multicolumn{5}{|l|}{ Unilateral or bilateral } \\
\hline Left fracture right thrombus & 22 & - & 22 & \multirow{5}{*}{$\begin{array}{l}\leq \\
0.001\end{array}$} \\
\hline Right fracture left thrombus & 34 & - & 34 & \\
\hline Left fracture bilateral thrombosis & 50 & - & 50 & \\
\hline Right fracture bilateral thrombosis & 34 & - & 34 & \\
\hline Only affected limb thrombosis & - & 333 & 333 & \\
\hline No lower extremities thrombosis & - & 981 & 981 & \\
\hline \multicolumn{5}{|l|}{ Types of fracture } \\
\hline Above the knee & 109 & 812 & 921 & \multirow[t]{3}{*}{0.001} \\
\hline Around the knee & 14 & 264 & 278 & \\
\hline Below the knee & 17 & 238 & 255 & \\
\hline \multicolumn{5}{|l|}{ Types of venous thrombosis } \\
\hline central & 1 & 8 & 9 & \multirow{3}{*}{$\leq .001$} \\
\hline peripheral & 131 & 289 & 420 & \\
\hline mixed & 8 & 36 & 44 & \\
\hline \multicolumn{5}{|l|}{ Medical morbidity } \\
\hline Hypertension (\%) & $46(32.86)$ & $288(21.91)$ & 334 & 0.003 \\
\hline Diabetes (\%) & $18(12.86)$ & $106(8.07)$ & 124 & 0.054 \\
\hline Coronary heart disease (\%) & $46(32.86)$ & $286(21.76)$ & 332 & 0.003 \\
\hline Arrhythmia (\%) & 19 (13.57) & $142(10.81)$ & 161 & 0.322 \\
\hline
\end{tabular}

ASA, American Society of Anesthesiologists; CRP, C- reactive protein. 


\begin{tabular}{|c|c|c|c|c|}
\hline & Thrombosis & $\begin{array}{l}\text { No } \\
\text { Thrombosis }\end{array}$ & Overall & $P$ \\
\hline Stroke (\%) & $12(8.57)$ & $76(5.78)$ & 88 & 0.189 \\
\hline Associated trauma (\%) & $14(10.00)$ & $167(12.71)$ & 181 & 0.356 \\
\hline BMI & $\begin{array}{l}21.15 \pm \\
7.64\end{array}$ & $22.41 \pm 5.31$ & $\begin{array}{l}22.28 \pm \\
5.58\end{array}$ & 0.331 \\
\hline $\begin{array}{l}\text { Days between fracture and hospitalization } \\
\text { (days) }\end{array}$ & $\begin{array}{l}6.14 \pm \\
28.49\end{array}$ & $2.05 \pm 5.58$ & $\begin{array}{l}2.44 \pm \\
10.36\end{array}$ & 0.093 \\
\hline Days between fracture and operation (days) & $\begin{array}{l}8.01 \pm \\
42.35\end{array}$ & $6.84 \pm 12.77$ & $\begin{array}{l}6.95 \pm \\
17.86\end{array}$ & 0.744 \\
\hline \multicolumn{5}{|l|}{ ASA classification } \\
\hline 1 & 10 & 245 & 255 & \multirow[t]{4}{*}{0.005} \\
\hline 2 & 88 & 766 & 854 & \\
\hline 3 & 41 & 299 & 340 & \\
\hline 4 & 1 & 4 & 5 & \\
\hline \multicolumn{5}{|l|}{ Serum markers } \\
\hline D-Dimer at admission (mg/L) & $\begin{array}{l}13.93 \pm \\
13.91\end{array}$ & $12.58 \pm 20.33$ & $\begin{array}{l}12.71 \pm \\
19.80\end{array}$ & 0.453 \\
\hline D-Dimer at preoperation (mg/L) & $5.96 \pm 6.27$ & $5.05 \pm 6.32$ & $5.15 \pm 6.31$ & 0.289 \\
\hline CRP at admission (mg/L) & $\begin{array}{l}21.83 \pm \\
34.47\end{array}$ & $21.14 \pm 31.96$ & $\begin{array}{l}21.11 \pm \\
32.22\end{array}$ & 0.868 \\
\hline
\end{tabular}


Table 2

Patient characteristics according to postoperative ultrasound

\begin{tabular}{|c|c|c|c|c|}
\hline & Thrombosis & $\begin{array}{l}\text { No } \\
\text { Thrombosis }\end{array}$ & Overall & $P$ \\
\hline Number & 295 & 1159 & 1454 & \\
\hline Age & $71.76 \pm 13.42$ & $59.62 \pm 19.66$ & $62.09 \pm 19.19$ & $\leq .501$ \\
\hline \multicolumn{5}{|l|}{ Gender } \\
\hline Female & 191 & 601 & 792 & \multirow{2}{*}{$\leq .001$} \\
\hline Male & 104 & 558 & 662 & \\
\hline \multicolumn{5}{|l|}{ Unilateral or bilateral } \\
\hline Left fracture right thrombus & 25 & - & 25 & \multirow{5}{*}{$\leq .001$} \\
\hline Right fracture left thrombus & 33 & - & 33 & \\
\hline Left fracture bilateral thrombosis & 127 & - & 127 & \\
\hline Right fracture bilateral thrombosis & 110 & - & 110 & \\
\hline Only affected limb thrombosis & - & 433 & 433 & \\
\hline No lower extremities thrombosis & - & 726 & 726 & \\
\hline \multicolumn{5}{|l|}{ Types of fracture } \\
\hline Above the knee & 240 & 681 & 921 & \multirow{3}{*}{$\leq .001$} \\
\hline Around the knee & 34 & 244 & 278 & \\
\hline Below the knee & 21 & 234 & 255 & \\
\hline \multicolumn{5}{|l|}{ Types of venous thrombosis } \\
\hline central & 3 & 10 & 13 & \multirow{3}{*}{$\leq .001$} \\
\hline peripheral & 281 & 377 & 658 & \\
\hline mixed & 11 & 46 & 57 & \\
\hline \multicolumn{5}{|l|}{ Medical morbidity } \\
\hline Hypertension (\%) & $85(28.81)$ & $249(21.48)$ & 334 & 0.009 \\
\hline Diabetes (\%) & $32(10.85)$ & $92(7.94)$ & 124 & 0.120 \\
\hline Coronary heart disease (\%) & 87 (29.49) & $245(21.14)$ & 332 & 0.003 \\
\hline Arrhythmia (\%) & $41(13.90)$ & $120(10.35)$ & 161 & 0.091 \\
\hline
\end{tabular}

ASA, American Society of Anesthesiologists; CRP, C- reactive protein. 


\begin{tabular}{|c|c|c|c|c|}
\hline & Thrombosis & $\begin{array}{l}\text { No } \\
\text { Thrombosis }\end{array}$ & Overall & $P$ \\
\hline Stroke (\%) & $27(9.15)$ & $61(5.26)$ & 88 & 0.017 \\
\hline Associated trauma (\%) & $34(11.53)$ & $147(12.68)$ & 181 & 0.588 \\
\hline BMI & $21.90 \pm 6.58$ & $22.38 \pm 5.30$ & $22.28 \pm 5.58$ & 0.516 \\
\hline Length of hospital & $9.76 \pm 3.84$ & $9.54 \pm 4.26$ & $9.58 \pm 4.18$ & 0.429 \\
\hline $\begin{array}{l}\text { Days between fracture and } \\
\text { hospitalization (days) }\end{array}$ & $3.49 \pm 19.85$ & $2.18 \pm 5.85$ & $2.44 \pm 10.36$ & 0.263 \\
\hline $\begin{array}{l}\text { Days between fracture and operation } \\
\text { (days) }\end{array}$ & $6.98 \pm 29.78$ & $6.95 \pm 13.23$ & $6.95 \pm 17.86$ & 0.986 \\
\hline \multicolumn{5}{|l|}{ ASA classification } \\
\hline 1 & 21 & 234 & 255 & \multirow{4}{*}{$\leq .001$} \\
\hline 2 & 183 & 671 & 854 & \\
\hline 3 & 89 & 251 & 340 & \\
\hline 4 & 2 & 3 & 5 & \\
\hline Duration of operation (mins) & $\begin{array}{l}109.39 \pm \\
57.43\end{array}$ & $\begin{array}{l}113.68 \pm \\
73.00\end{array}$ & $\begin{array}{l}112.81 \pm \\
70.12\end{array}$ & 0.349 \\
\hline Blood loss (ml) & $\begin{array}{l}293.39 \pm \\
300.20\end{array}$ & $\begin{array}{l}223.05 \pm \\
241.20\end{array}$ & $\begin{array}{l}237.32 \pm \\
255.74\end{array}$ & $\leq .001$ \\
\hline Liquid transfusion (ml) & $\begin{array}{l}1730.61 \pm \\
623.94\end{array}$ & $\begin{array}{l}1739.41 \pm \\
557.11\end{array}$ & $\begin{array}{l}1737, .62 \pm \\
571.12\end{array}$ & 0.814 \\
\hline \multicolumn{5}{|l|}{ Serum markers } \\
\hline D-Dimer at admission (mg/L) & $16.46 \pm 23.89$ & $11.76 \pm 18.52$ & $12.71 \pm 19.80$ & 0.002 \\
\hline D-Dimer at preoperation $(\mathrm{mg} / \mathrm{L})$ & $6.10 \pm 6.17$ & $4.90 \pm 6.33$ & $5.15 \pm 6.31$ & 0.072 \\
\hline D-Dimer at postoperative 1 day (mg/L) & $10.29 \pm 9.02$ & $5.93 \pm 6.25$ & $6.85 \pm 7.15$ & $\leq$ \\
\hline D-Dimer at postoperative 3 day (mg/L) & $7.69 \pm 6.97$ & $5.16 \pm 6.03$ & $5.73 \pm 6.34$ & $\leq_{0.001}$ \\
\hline D-Dimer at postoperative 5 day (mg/L) & $10.56 \pm 7.47$ & $6.20 \pm 5.04$ & $7.27 \pm 6.02$ & $\leq .001$ \\
\hline CRP at admission (mg/L) & $22.14 \pm 33.96$ & $21.00 \pm 31.80$ & $21.22 \pm 32.22$ & 0.731 \\
\hline CRP at postoperative 5 day (mg/L) & $36.68 \pm 39.47$ & $32.49 \pm 44.19$ & $33.33 \pm 43.37$ & 0.437 \\
\hline
\end{tabular}




\section{Figures}

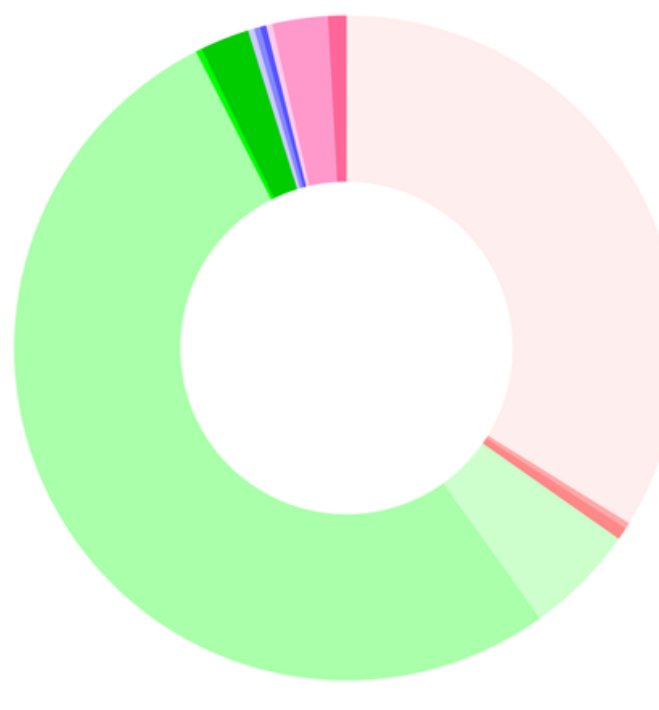

Total $=334$
$33.83 \%$ From No to peripheral

$0.30 \%$ From No to central

$0.60 \%$ From No to mixed

$5.39 \%$ From peripheral to No

$52.40 \%$ From peripheral to peripheral

$0.30 \%$ From peripheral to central

$2.40 \%$ From peripheral to mixed

$0.30 \%$ From central to peripheral

$0.30 \%$ From central to central

$0.30 \%$ From central to mixed

$0.30 \%$ From mixed to No

$2.69 \%$ From mixed to peripheral

$0.90 \%$ From mixed to mixed

\section{Figure 1}

The dynamic changes of uninjured limb DVT before and after operation. 